\title{
Angioinvasive Aspergillosis of the Central Nervous System
}

\author{
Aasef G. Shaikh, Sophia Sundararajan
}

Keywords: embolism, immunocompromised, infarction

Aspergillosis is usually seen in immunocompromised patients, but it may also occur in immunocompetent individuals. Invasion of intravascular aspergillus fungus hyphae in the central nervous system (CNS) causes multiple embolic infarcts. These organisms then erode the blood vessel, leading to hemorrhagic transformation, purulent necrosis, and lesion expansion without edema. ${ }^{1-3}$ Aspergillosis in the CNS has a peculiar radiological presentation. Exemplary MRI features suggestive of CNS aspergillosis are described here in a 50-year-old immunocompromised woman who was evaluated for altered awareness (Figure 1).

The arrows in Figure 1A depict focal hyperintensity in diffusion-weighted imaging (DWI) and the hypointense correlation on apparent diffusion coefficient (ADC). Larger lesions had a core with a hypointense signal on DWI and a bright signal on ADC (Figure 1A, open arrows). The lesions were isointense on $\mathrm{T} 1$, but hyperintense on a fluid-attenuated inversion recovery sequence (Figure 1A, arrow). There was no contrast enhancement, edema, or midline shift. These findings suggested acute infarction with a purulent core, which is a possible manifestation of aspergillosis. Similar lesions affected multiple brain regions as noted in the MRI performed on day one (Figure 1B, top panel). The lesions were more abundant on day four, and the preexisting lesions expanded in size (Figure 1B, bottom panel). This constellation of MRI findings is typical for CNS aspergillosis. ${ }^{2,3}$ Autopsy confirmed disseminated aspergillosis of the brain, lung, and thyroid (Figure 1C).
A differential diagnosis for such presentation includes metastatic tumors, multiple infarcts resulting from septic bacterial emboli, or embolic shower from a cardiac thrombus. Rapid progression, as seen in our patient, is not typical for metastatic tumors, but sometimes it is difficult to differentiate abscess resulting from aspergillosis from that resulting from a neoplastic process. Ischemic infarcts from cardiogenic emboli may increase in number and involve spatially independent locations, but they do not increase in size, except in cases in which ischemic penumbra is converted to a complete infarct. Hemorrhagic conversion of an ischemic lesion resulting from septic bacterial emboli is also possible, but malignant expansion as seen in aspergillosis is less likely.

\section{ACKNOWLEDGMENTS AND FUNDING}

Authors have no disclosures.

\section{REFERENCES}

1. Beal MF, O'Carroll CP, Kleinman GM, et al. Aspergillosis of the nervous system. Neurology. 1982;32:473-9.

2. Miaux Y, Ribaud P, Willams M, et al. MR of cerebral aspergillosis in patients who have had bone marrow transplantation. Am J Neuroradiol. 1995;16:555-62.

3. Starkey J, Moritani T, Kirby P. MRI of CNS fungal infections: review of aspergillosis to histoplasmosis and everything in between. Clin Neuroradiol. 2014;24:217-30. 


\section{A}

Embolic infarct with necrotic core without edema or midline shift

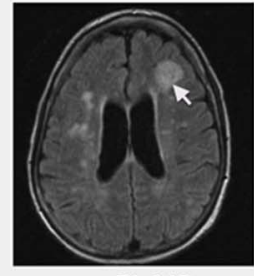

FLAIR

Fluid Attenuated Inversion Recovery

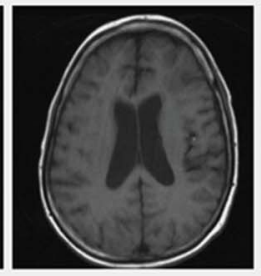

$\mathrm{T} 1$

weighted

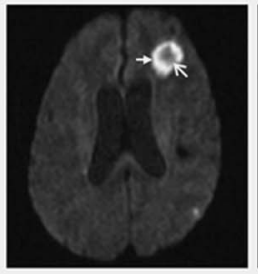

DWI

Diffusion Weighted Imaging

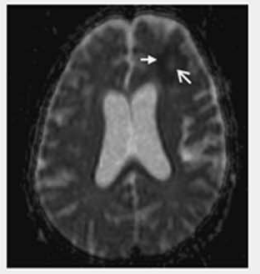

$\mathrm{ADC}$

Automated Diffusion Coefficient

B

Fulminant expansion of multiple embolic infarcts

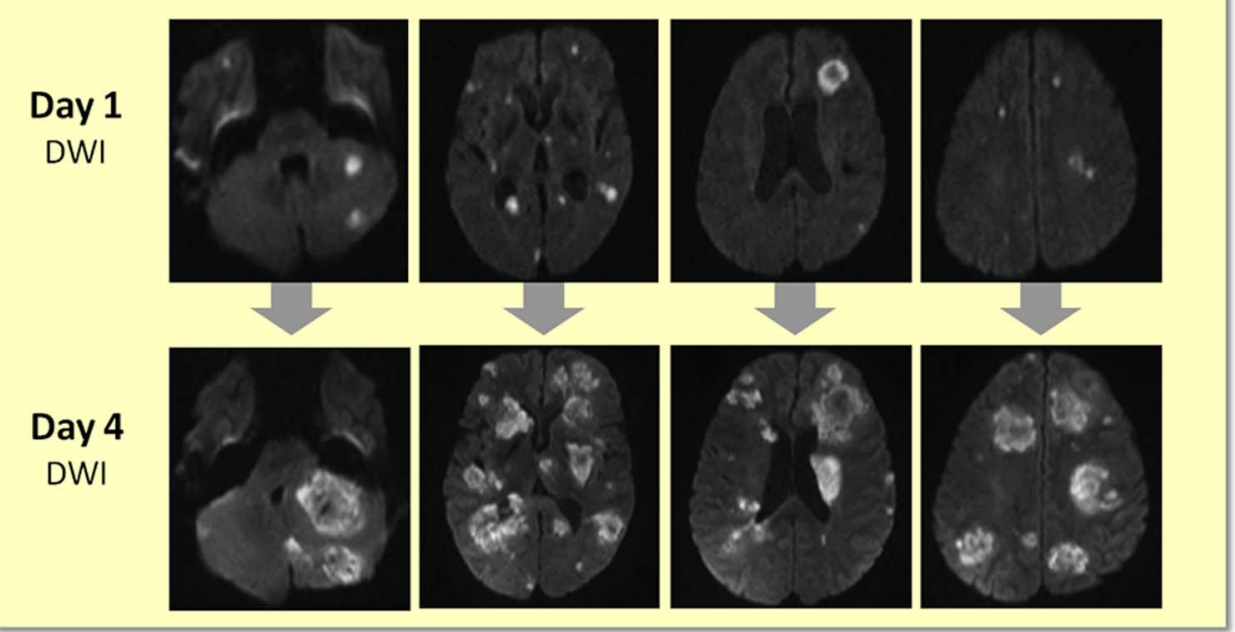

C

Histology showing disseminated Aspergillus Fumigatus hyphae (arrows)
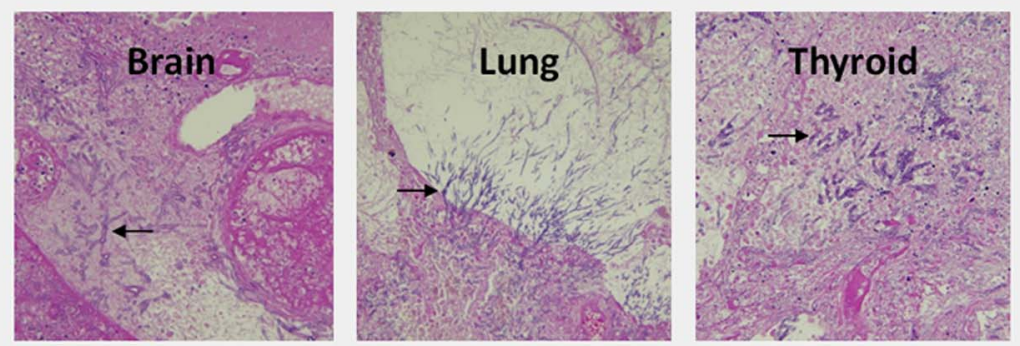

Figure 1: MRI and histological findings of CNS aspergillosis in an immunocompromised woman. (A) Magnetic resonance imaging features of CNS aspergillosis as seen with focal hyperintensity in DWI and fluid-attenuated inversion recovery (FLAIR), but hypointensity in ADC (arrows). Closed white arrows show areas of ischemia, whereas open white arrows depict the hemorrhagic core. Ischemic lesions are not discrete in a noncontrast T1-weighted sequence. (B) Fulminant expansion of the lesions within a short (four-day) period. Lesions affected multiple brain regions as noted in the MRI scan performed on day one (top panel). The lesions were more abundant on day four, and preexisting lesions expanded in size (bottom panel). (C) Histology of brain, lung, and thyroid tissues confirmed disseminated aspergillosis. Closed black arrows depict aspergillus fumigatus hyphae. 